\title{
LOA-CAST: a novel low-overhead information broadcast scheme for vehicular ad hoc networks
}

\author{
Wen-Hsing Kuo ${ }^{*}$ and Shun-Hsung Wu
}

\begin{abstract}
In this paper, we propose a novel scheme for broadcasting non-urgent information in vehicular ad hoc networks (VANETS). The scheme, called low-overhead aggregated broadcast (LOA-CAST), aggregates information from different sources on a road and forwards it periodically. By aggregating the information from different sources and periodically propagating the message, it reduces the overall signaling overhead and dynamically adjusts the propagation distance of information according to the source density. We use analysis to evaluate the behavior of LOA-CAST in the presence of malicious/malfunctioning nodes. The results show that LOA-CAST can still function well and control the overall overhead if such nodes exist. Moreover, the results of simulations demonstrate that LOA-CAST incurs a reasonable overhead while propagating information efficiently.
\end{abstract}

Keywords: Vehicular networks; Broadcast; Protocol; Signaling overhead

\section{Introduction}

A vehicular ad hoc network (VANET) [1] is a special type of mobile ad hoc network that enables vehicles to communicate with each other as well as with roadside units in the vicinity. With this technology, many traffic-related applications, such as accident warning systems, congestion detection schemes, and traffic-related information broadcasts, can be implemented to provide more comfortable driving experiences. Each vehicle in a VANET must be equipped with a wireless interface, which typically operates under the IEEE 802.11 Wi-Fi standard [2]. In such a network, the information to be transmitted usually has different degrees of urgency. Some transmissions (e.g., emergency/accident notifications or QoS-sensitive multimedia connections) require short delays and low loss rates, so they should be given a higher priority than non-urgent messages. The latter provides drivers with information about the local environment (e.g., weather conditions, the average speed of traffic on a particular road, the number of vacant spaces in a parking lot, and advertisements from stores). For these kinds of messages, a

* Correspondence: whkuo@saturn.yzu.edu.tw

Department of Electrical Engineering, Yuan-Ze University, Taoyuan 320,

Taiwan

\section{Springer}

(c) 2014 Kuo and Wu; licensee Springer. This is an Open Access article distributed under the terms of the Creative Commons Attribution License (http://creativecommons.org/licenses/by/2.0), which permits unrestricted use, distribution, and reproduction in any medium, provided the original work is properly credited. certain degree of delay is tolerable, and their transmission can be paused, or even postponed indefinitely, if bandwidth is scarce.

Basically, drivers can obtain traffic-related information in two ways: actively and passively. In the active approach, a vehicle sends queries to nodes in the area of interest and waits for the replies; in the passive approach, a vehicle simply receives traffic-related messages from different sources. For non-urgent environmental information, which we focus on in this paper, passive transmission makes more sense for two reasons. The first is the overhead issue. If several vehicles request the same information, the source node has to send separate replies containing identical messages, which wastes the radio bandwidth. The second reason is the blindness issue. Drivers may not be aware of information that may be of interest, e.g., a newly opened parking lot or gasoline station, and it is unlikely that they would request such information actively.

Although broadcasting environmental information is more reasonable, many technical issues must be addressed. The first is the management issue. Because information sources located in different positions broadcast messages independently, they use different 
relay nodes (i.e., the nodes that help forward messages); therefore, managing the total overhead of broadcasting services is a challenging task. It is very difficult for the relay nodes of different sources to perceive and negotiate with each other. When too many nodes broadcast information simultaneously, or there are malicious nodes that constantly send empty packets, the whole network may be overwhelmed. The second issue is related to efficiency. Messages containing local environmental information (e.g., the average speed on the current section of road or the number of vacant spaces in a parking lot) are relatively short. If each short message is broadcast separately, it may take longer for nodes to access the medium (e.g., sending RTS/CTS messages, waiting for DIFS, and even collisions with other nodes) than to broadcast the message body. However, if information from different sources could be aggregated and relayed as one larger message, the transmission time required by the access process could be reduced substantially. The third issue involves the flexibility of transmitting messages. Because of the number of time-varying broadcast sources and the dynamic total overhead, the broadcast range of each source should be adaptive. That is, the range should be extended to notify as many cars as possible when the radio resource allows, and limited when the traffic is heavy and the resource is scarce.

A number of position-based routing schemes designed to transmit data in static or dynamic topologies of VANETs have been proposed, e.g., [3,4], and some cluster-based routing schemes have also been designed, e.g., $[5,6]$. With these routing approaches and some additional design on higher layers, an active query service can be implemented. For example, Dikaiakos et al. [7] designed an integral service that enables drivers to actively query a source node whose position is known and obtain the latest information.

Meanwhile, several works have studied broadcast schemes in VANETs. Torrent-Moreno et al. [8] investigated the probability of successfully receiving broadcast data at a certain distance from the sender, Zhao et al. [9] proposed a scheme that enables cars or roadside units (RSUs) at an intersection to cache and forward data to improve the reliability of transmissions, and Tonguz et al. [10] designed a scheme that can function in different traffic conditions. However, these works focus on relaying and routing issues in single broadcast traffic. They do not consider the trouble a malicious node could cause or find ways to manage and reduce the broadcasting overhead.

The geocast paradigm is also important. Schemes based on such model, e.g., [11-14], broadcast information in a range, called a zone of relevance (ZOR), instead of to a predetermined group of nodes. In [13], Maihöfer et al. proposed a special type of geocast scheme that allows packets to remain in a range for a certain period of time so that all vehicles that come within the range during that period can receive the information. With this class of technologies, a 'virtual road sign' can be formed. These approaches may be effective for informing vehicles in a small range, but they are unsuitable for large-scale broadcasts because each message has fixed transmission range and is relayed separately. Hence, the range of the recipients cannot be dynamically adjusted to adapt to the network's traffic load. Moreover, because each source broadcasts separately over a wide area, the total overhead may be heavy and difficult to manage. Some researchers have also highlighted the importance of data aggregation. Authors in [14] propose a cluster-based scheme which provides drivers the forward visibility of $1.6 \mathrm{~km}$. The scheme works only on a straight road and does not consider the road topology such as branches and intersections. Dietzel [15] discusses a generic model of VANET data aggregation protocols. Like [14], he focuses on the aggregation and computation among 'homogeneous data,' (such as aggregating speed information of each car to find the overall traffic conditions). However, we focus on putting various kinds of information into a general-purpose broadcast mechanism to reduce and control the overall broadcasting overhead.

In this paper, we propose a scheme called lowoverhead aggregated broadcast (LOA-CAST), which is designed to distribute non-urgent environmental information via a best-effort approach. The scheme can deliver such information to vehicles several kilometers away from the source, address all the broadcasting issues mentioned earlier, and manage the total overhead. Through analysis, we show that the operation of the whole network is stable even if there are malfunctioning/malicious nodes in the network. Furthermore, the results of simulations show that the operations are robust and the signaling overhead is light.

The remainder of this paper is structured as follows. In Section 2, we introduce the system model and describe the operation of LOA-CAST. We also show that LOA-CAST is robust in the presence of malfunctioning/malicious nodes. In Section 3, we discuss the simulations conducted to evaluate the scheme's performance. Section 4 contains some concluding remarks.

\section{LOA-CAST scheme}

In the following subsections, we introduce the assumptions in which LOA-CAST is based on and the structure of the broadcast messages, discuss the scheme's architecture, describe the operation of each independent 
node, explain how messages from different sources are aggregated, and consider the robustness and signaling overhead.

\subsection{Assumptions and message contents}

LOA-CAST is based on three assumptions:

1. Each vehicle knows its current speed, position, and direction.

2. A vehicle is aware of the position and direction of each of its neighbors (i.e., those within its transmission range).

3. The clock of each vehicle is loosely synchronized.

These assumptions are realistic and are currently used by most VANET unicast and broadcast schemes, such as those discussed in the previous section. They are practical if every node is equipped with a Global Positioning System (GPS) device and periodically broadcasts its position to adjacent nodes.

To notify cars nearby, each information source sent broadcast messages (BMs) that are structured as follows:

- Time - the time the information is broadcast

- Duration (optional) - the longest period that a message should remain in the network

- Source ID - the ID of the information source

- Source position/direction - the direction and position of the sender

- Message body - the information each sender broadcasts

To illustrate, we consider an RSU setup near a parking lot to broadcast information about vacant spaces. Normally, a BM contains just one message entry from a single source. The structure of such a BM is shown below. However, when a number of messages are to be aggregated, BMs may contain multiple information entries. We discuss this aspect later in the paper.

Information structure of a BM

\subsection{Operations of the LOA-CAST scheme}

To aggregate BMs into the aggregated messages and forward them in a distributed manner, the operation of each node should be carefully designed. Therefore, we let each node operate in one of four modes basing on the received messages and current conditions. The four modes are silent mode, beating mode, source mode, and relaying mode. Figure 1 shows an example of the LOA-CAST scheme's operation in a single lane of traffic. Note that the cars in the opposite lane do not participate in the transmission illustrated here since they are responsible for forwarding information in another direction. When a car is at the head of a line and cannot receive messages from forward nodes, it should become the start of the transmission and initiate the aggregated messages for the cars behind. Therefore, it switches to the beating mode and periodically transmits a blank aggregated broadcast message $(\mathrm{ABM})$ to the nodes behind it. The ABM records the BMs of different sources that it passes as well as information about the assigned relay(s) in the next hop(s). On receipt of the $A B M$, each node that wants to broadcast a message (i.e., information source) transmits its BM. Then, the chosen relay integrates the BMs received in its transmission range and purges the message entries whose transmission duration has expired when forming the new ABM. The new ABM is then transmitted to the nodes behind the relay. By each relayer's repeating this operation continuously, all messages can be placed into the ABMs and propagated efficiently against the vehicles' directions.

Next, we detail the operations of the four modes.

Beating mode. If a car does not receive an ABM from forward nodes for a certain period of time, it should initiate the periodical transmission and transmit an $\mathrm{ABM}$ to the nodes behind it. Therefore, it switches to beating mode and broadcasts a blank ABM periodically. In addition, basing on the position of the neighbor nodes, the beater finds the most distant node(s) on each branch to act as the next hop relay(s) and assigns them in the ABM accordingly. The relays are responsible for distributing the ABM to the next hop.

Source mode. On receipt of an ABM, a source broadcasts a BM. To ensure that different kinds of information are propagated effectively, LOA-CAST exploits three types of

$<$ time $>15: 4503$ s $</$ time $>$

$<$ duration $>10$ minutes $<$ /duration $>$

$<$ SenderID $>$ XXXXXXXX $<$ SenderID $>$

//the MAC address of the RSU

$<$ position $>$ avenue $13 / 2 \mathrm{~km}$ /east direction</position $>$

$<$ body $>$ parking lot/16 vacant spaces/1dollar per hour $</$ body $>$

//the information it wants to broadcast to drivers in the vicinity 


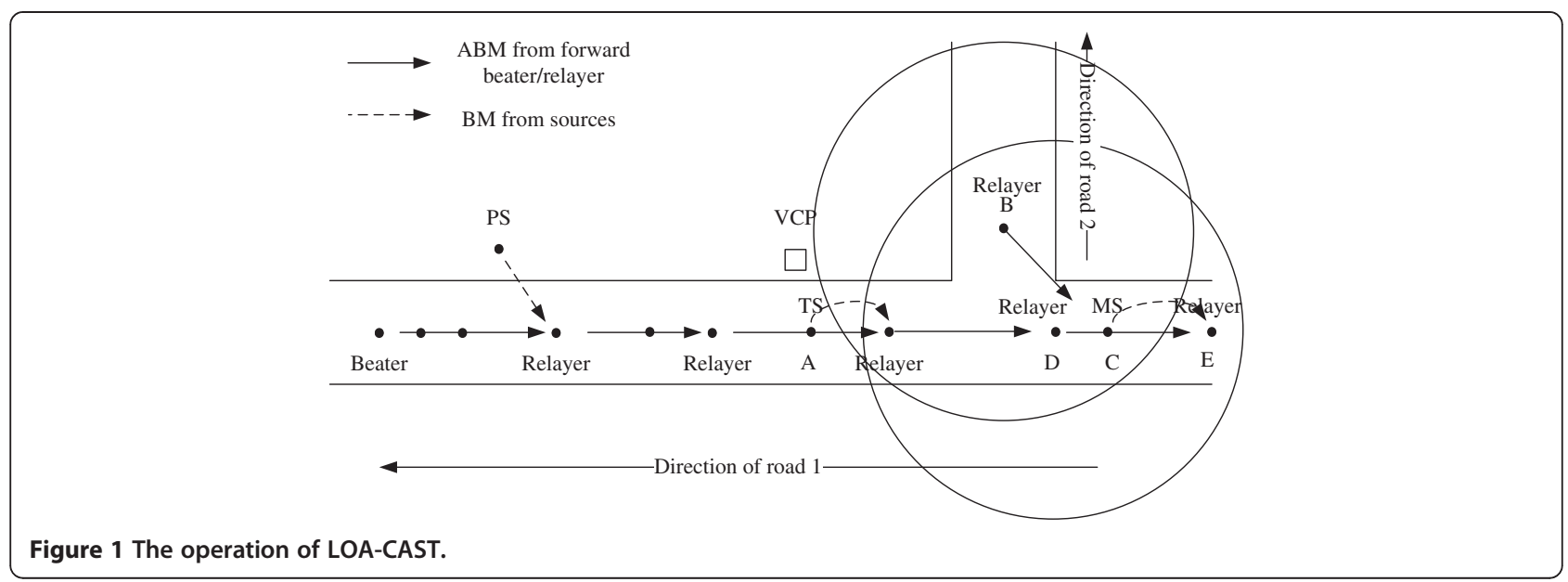

sources: permanent sources (PSs), temporary sources (TSs), and multiple sources (MSs). A PS is a node that belongs to a specific entity and broadcasts the entity's advertising messages, such as an RSU setup by a store or a parking lot. TSs report important information for areas that do not have dedicated PSs. To gather such information, 'virtual check points' (VCPs) can be predetermined and stored in each vehicle's GPS device. When an ABM whose range covers the VCP is broadcast, the closest vehicle to the VCP becomes the TS and is responsible for assessing and reporting local conditions. Since each car has the positions of its neighbor, it is feasible to know whether it is the closest one. The operations of PS and TS are illustrated in Figure 1. The only difference between PS and TS is that the latter becomes an ordinary (i.e., silent) node after transmitting a BM. Since the positions of vehicles are dynamic, when the next ABM comes, the TS of a VCP can be different. Finally, an MS handles multiple ABMs. If a road has an intersection, the different branches may propagate $\mathrm{ABMs}$ at the same time and generate a heavy traffic load, so the ABMs should be merged. Therefore, when a relayer receives another $\mathrm{ABM}$ before transmitting its own ABM, it becomes an MS and broadcasts its own $\mathrm{ABM}$ as a multiple-entry $\mathrm{BM}$ to let another ABM's relayer merge the information. As shown in Figure 1, when car $C$ receives the $\mathrm{ABM}$ from car $\mathrm{B}$, it becomes a relayer and counts down for an interval before forwarding the ABM. However, when it receives another ABM from D during the countdown, it becomes an MS and immediately transmits a BM containing all the entries in its ABM. As a result, relayer $E$ (which receives the $A B M$ from $D$ ) merges the messages from $C$ and transmits the new $A B M$ to the cars behind it. When there are multiple ABMs from different roads at an intersection, or a malicious node keeps sending a large number of ABMs, this mechanism can reduce the number of ABMs from different sources but still let them propagate once in every given period $T_{\text {period. }}$ In other words, it maintains the transmission rate and overhead. Irrespective of the source type (i.e., a PS, TS, or MS), it always transmits the BM upon receipt of the ABM. If there are multiple sources in an ABM's transmission area, existing approaches (e.g., carrier sense multiple access with collision avoidance (CSMA/CA)) can be used to avoid a collision. After transmitting the BM, a TS or MS becomes an ordinary node, but a PS remains in the source mode and transmits its BM when the next $\mathrm{ABM}$ is received.

Relaying mode. When an ABM is transmitted, the most distant node on each road branch within the transmission range becomes the relaying node and is responsible for forwarding the ABM again. After relaying the $\mathrm{ABM}$, it switches to a normal node. Like the beater, basing on its own neighbor information, a relayer decides the next hop's relayer and adds the ID to the transmitted $\mathrm{ABM}$. When the transmission range of the relayer covers crossroads, it selects a relayer on each road. To limit the bandwidth consumption, the size of the ABM is fixed. Therefore, when the number of entries reaches a given limit, the oldest entries (i.e., those with the earliest transmission time) are purged.

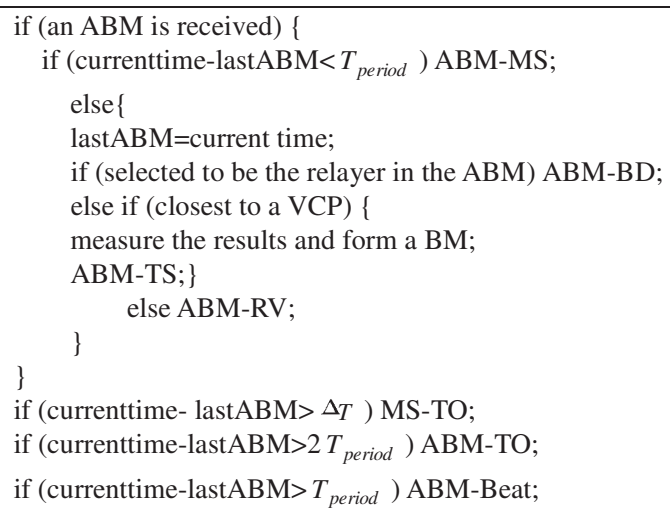

Figure $\mathbf{2}$ The event checking procedure of each node. 
Silent mode. When a node does not have to transmit or forward messages, it receives and decodes the ABMs to obtain the latest information.

Conclusively, basing on the four modes, the head of line on each road becomes a beater and pumps ABM periodically along the road, while each chosen relayer forwards the packets to help them propagate. As an ABM passes by, the information sources transmit the latest messages. Therefore, when operating correctly, each vehicle can acquire information from the ABMs it receives. The signaling overhead is reduced because of two reasons. First, unlike flooding approaches, duplicated ABMs are merged by MSs. Second, instead of being transmitted separately, the broadcast messages of all kinds of sources are integrated in LOA-CAST, and the process of accessing the transmission medium (i.e., conducting CSMA/CA) is substantially saved.

\subsection{State transition operations and clock settings in LOA-CAST}

The state transition operations of LOA-CAST are shown in Figures 2 and 3. Figure 2 shows the procedure that each node executes periodically to detect events, while Figure 3 shows the state transition diagram based on the occurring events. As shown in Figure 2, irrespective of the type of node, when it receives an ABM, the node first checks if the cooling-down interval of the previous $\mathrm{ABM}$ has expired. If it has not expired, an event ABM-MS is activated and a relaying node becomes an MS. Then, if it is selected as the new relayer in the received $A B M$, the event $A B M-B D$ is activated. Moreover, each node periodically checks the current locations of its neighbors and itself, as we explained in the assumptions. If the node is currently the closest node to a VCP upon receiving the ABM, the event ABM-VS is activated. However, if none of the above conditions are satisfied, the event ABM-RV is activated to switch a beater to a silent node.

In addition to receiving ABMs, a node also continuously checks the time to activate some events. For example, MS-TO (i.e., message time-out) represents the event that the previous ABM's interval has passed $\Delta T$, which means it is time for a relayer to transmit an ABM to the next hop. However, if an ABM has not been received for two $T_{\text {period }}$ ABM-TO (i.e., ABM time-out) is activated and the node is switched to a beater. Finally, the event ABM-Beat makes a beater initiate a blank ABM periodically.

Figure 3 shows the state transition diagram based on the detected events in Figure 2. When a node enters the network, it is in the silent mode by default. If the node does not receive an ABM from cars in front of it for two $T_{\text {period }}$ (i.e., event ABM-TO), it becomes a beater and

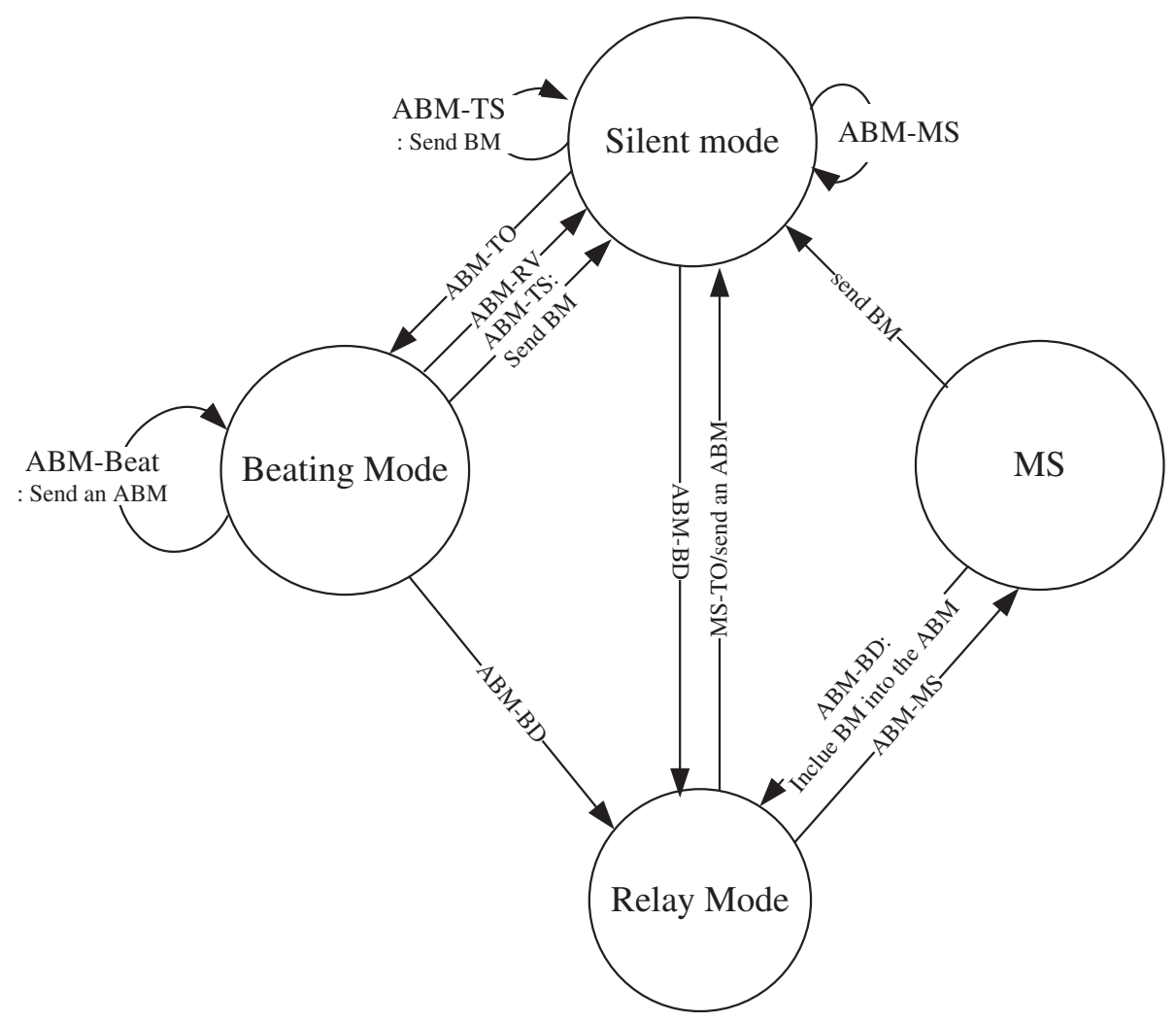

Figure 3 The state transition diagram of a normal node. 
periodically transmits an empty $\mathrm{ABM}$ to cars behind it every $T_{\text {period }}$ (i.e., $\left.A B M-B e a t\right)$. It becomes a normal node when it receives an ABM from cars in front of it. Next, each node switches to the relay mode when chosen as a relayer by an $\mathrm{ABM}$ (i.e., $\mathrm{ABM}-\mathrm{BD}$ ), transmits a new $\mathrm{ABM}$, and becomes silent after $\Delta t$ (i.e., event MS-TO occurs). When a relayer receives another $\mathrm{ABM}$ before transmitting its $\mathrm{ABM}$ (i.e., $\mathrm{ABM}-\mathrm{MS}$ ), it acts as an MS and immediately transmits its $A B M$ as a $B M$ and returns to silent mode.

For RSUs, which are always PSs, the operation is much simpler because they do not become beaters or relayers and do not switch to the silent mode. They simply transmit a BM whenever an ABM is received.

\subsection{The aggregation operation and the transmission interval between messages}

In this section, we consider the integration of information and the transmission interval between ABMs. Each relayer must aggregate all the information from different sources in the transmission range as well as that in received ABMs into a new ABM. Given the information entries from the $\mathrm{ABM}$ and $\mathrm{BMs}$, the relayer first removes those that have reached time-out (i.e., current time-time $>$ duration) in the $\mathrm{ABM}$ and those that have incorrect time tags (i.e., time > current time). This operation is necessary because a malicious RSU may use a longer time tag than the current time to extend the lifetime of its messages. Next, the relayer removes duplicate entries from the same source (i.e., messages with same ID) and only keeps the most recent one (i.e., the message with the largest time value). If the number of entries is still greater than the size of the ABM, the relayer keeps entries which have longer remaining lifetime and are from more approximate distance to the source so that the latest and closest information (i.e., more useful) can be forwarded. Under this aggregation approach, the propagation time of information and the transmission range can be adapted to the bandwidth condition. Given the size of the $\mathrm{ABM}$, the larger the number of messages, the sooner the message is full and the earlier message is removed from the ABM. Therefore, each message will have less time to be forwarded along the road and thus has propagated over a smaller range. Conversely, if there are fewer sources, each message can remain in the ABM for a longer period and be broadcast over a wider range. In other words, the transmission range is adaptive to the number of sources, while the transmission overhead of LOA-CAST is fixed.

Next, we discuss the transmission interval. After receiving an $A B M$ from the forward relayer/beater, each relayer should forward it as soon as possible to minimize the delay. However, the relayer must also wait for all the sources within range to transmit their latest information. We denote a relayer's waiting period as $\Delta T$. Because, each beater

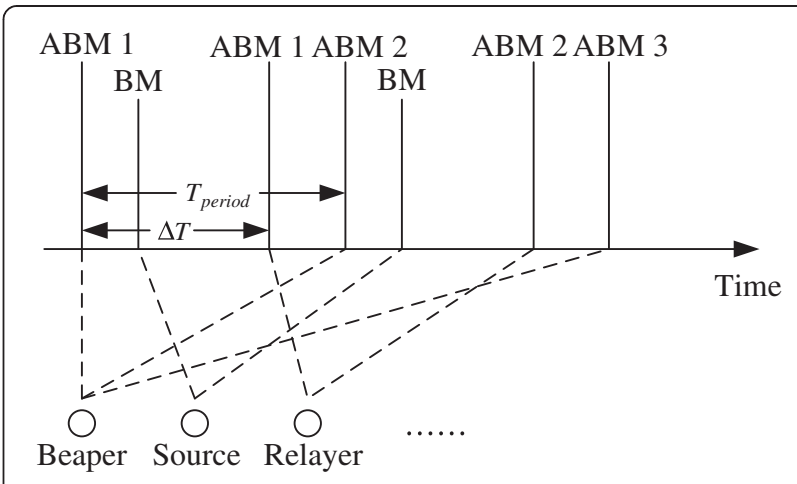

Figure 4 Transmission timeline of nodes.

transmits an ABM every $T_{\text {period, }}$ it is necessary that $0<\Delta T \leq T_{\text {period }}$ so that each ABM can be forwarded before the next one passes by. The transmission time of adjacent nodes is shown in Figure 4, where ABM $x$ denotes the $x$ th $\mathrm{ABM}$ sequence initiated by the beater. The sources within the relayer's transmission range transmit one BM before the relayer forwards the ABM (i.e., before $\Delta T$ ). The values of $\Delta T$ and $T_{\text {period }}$ should be set according to the preferred signaling overhead and the information speed. A large $\Delta T$ setting may reduce the transmission speed, while a very small setting would not allow sufficient time for sources to transmit information.

\subsection{Robustness of LOA-CAST}

In this section, we discuss the robustness of LOA-CAST and show that it can limit the signaling overhead caused from malfunctioning/malicious nodes. We consider a section of a road where some nodes are not operating correctly and study the behavior of the nodes behind them (i.e., those that may be affected). Because LOA-CAST only uses BMs and ABMs, we consider three types of malicious behavior: (1) a passive attack, where nodes disrupt the operation of LOA-CAST by not sending necessary BMs and ABMs; (2) an active BM attack, which tries to paralyze the network by flooding it with BMs; and (3) an active ABM attack. Figure 5 shows the analysis scenario.

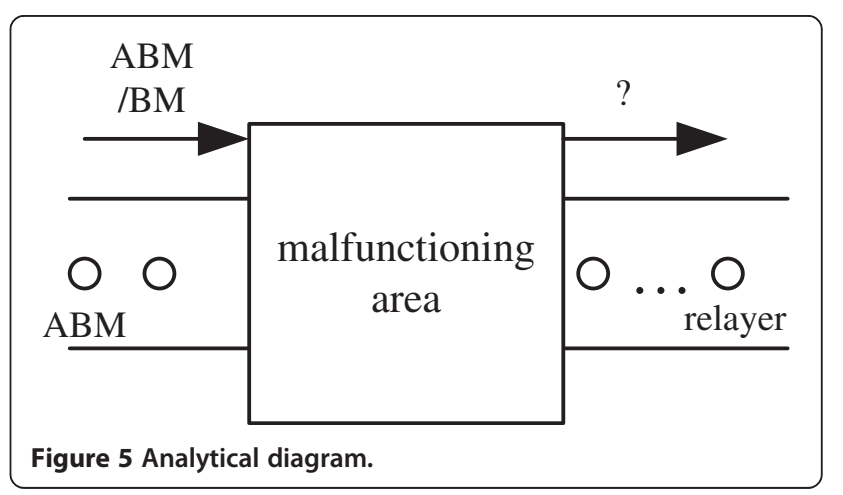


In a passive attack, if a node does not receive ABMs

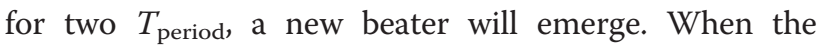
node receives an $\mathrm{ABM}$ from the forward direction, it will become a normal node again. Therefore, although a passive attack of a node which is supposed to be the relayer may block the information transmitted by nodes in front of the vehicle, it will not affect the operation of nodes and information sent by sources behind the vehicle. In an active BM attack, malicious nodes transmit numerous BMs that may block the space in ABMs and replace the information sent by sources in front of the target node. However, it does not affect the transmission of sources behind the attacked node because they always have later timestamps and can overwrite the previous BMs. Therefore, BMs behind the attacked node are not affected and can always be merged with an ABM. Even if the malicious node forges the timestamp so that it is less than the current time in the BM, the message can be detected easily and removed by the relayer. Finally, in an active ABM attack, a large number of ABMs are transmitted from a malfunctioning area, and several relayers may be responsible for forwarding them. When the interval between the ABMs is less than the $T_{\text {period }}$, nodes will be switched to the 'cooling-down' period, which will reduce the transmission rate of the ABMs.
If a malicious node is the only node that can relay messages on a road, it is inevitable that it will block forwarded messages; however, the broadcasts and operation of the nodes behind the attacked node are not affected. Each source and relayer after the attacked source can still operate and forward information normally.

\section{Performance evaluation}

The detailed setting of the simulations is as follows. We first use MOVE simulator to construct road topology and generate node movement. Then, we used NS-2 to evaluate the transmission performance. The two road models are designed as shown in Figure 6a,b. Model I is a 2,000-m single-lane road designed to simulate a highway, and model II is a grid road model of an urban environment. The speed limit of the first model is set as $110 \mathrm{~km} / \mathrm{h}$, and there are several equidistant tollbooths on the road. Cars decelerate to zero when approaching a booth and accelerate after passing through it. An RSU is placed at the midpoint of the road. In model II, the speed limit is $40 \mathrm{~km} / \mathrm{h}$, and there are traffic lights at each intersection. The network settings are as follows: The transmission range of each vehicle is $250 \mathrm{~m}$, the messages are transmitted in UDP packets, the payload size of an $A B M$ is set at $1 \mathrm{~KB}$ and that of a BM is set at $0.5 \mathrm{~KB}$, and the values of $T_{\text {period }}$ and $\Delta T$ are 5 and $1 \mathrm{~s}$, respectively. In both simulations,

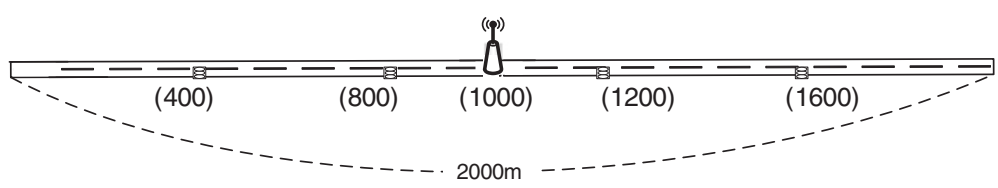

(a)

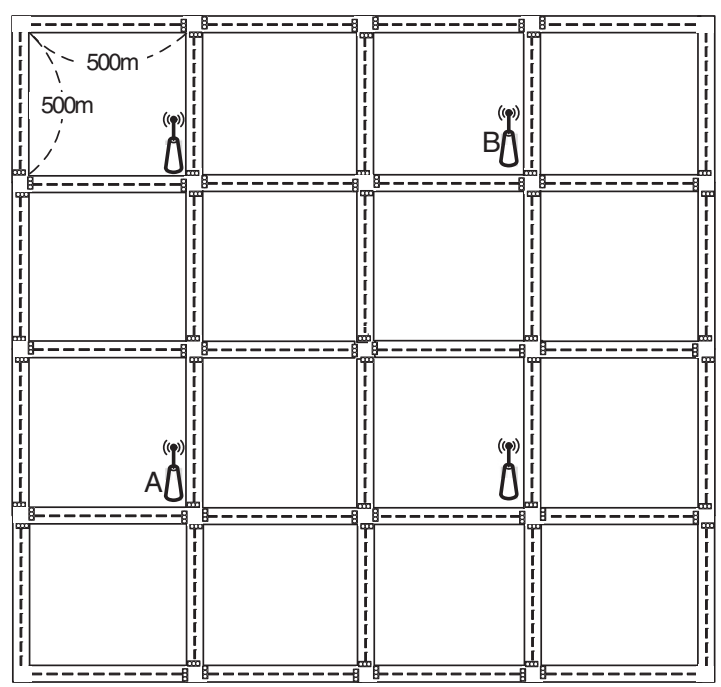

(b)

Figure 6 Simulation environment. (a) Model I. (b) Model II. 
the cars are generated and placed at the given spot (i.e., the start of the road in model I and the four corners in model II) and vanished when they reach the margin of the environment (i.e., the end of the road in model I and the four corners in model II). To evaluate the impact of the vehicle density, we adjust the number of cars entering the environment per second. Also, for each setting, the simulation lasts for $1,500 \mathrm{~s}$, and the results are averaged.

\subsection{Model I}

We observe the signaling overhead and propagation speed of messages in model I and study the impact of the vehicle density and the positions of the tollbooths. In this work, the signaling overhead is defined as the average amount of data each car receives per second, which refers to the amount of time the proposed scheme occupies in each car's transmission medium. On the other hand, the propagation speed is determined by calculating the time required for an $\mathrm{ABM}$ to be forwarded from the RSU to the end of the road. In the simulations, we adjust the entry frequency of cars as well as the number of tollbooths, which are placed at equal distances on the road.

Figure 7 shows LOA-CAST's performance in the highway scenario. The results in Figure 7a demonstrate that regardless of the number of tollbooths, the signaling overhead and the propagation time maintain similar trends, meaning that the behavior of LOA-CAST is stable under different densities and traffic conditions of vehicles. The reason is that in every $T_{\text {period }}$, each vehicle receives approximately two ABMs from relayers in front and behind it, irrespective of the traffic pattern. The average signaling overhead perceived by users is less than $1.4 \mathrm{~KB} / \mathrm{s}$, which is relatively light and acceptable for general Wi-Fi transmissions. In Figure $7 \mathrm{~b}$, the propagation delay of ABMs is higher when the entry rate is between 0.17 and 0.24 cars per second. This is because when the traffic is light, the

Signaling Overhead vs. Vehicle Density

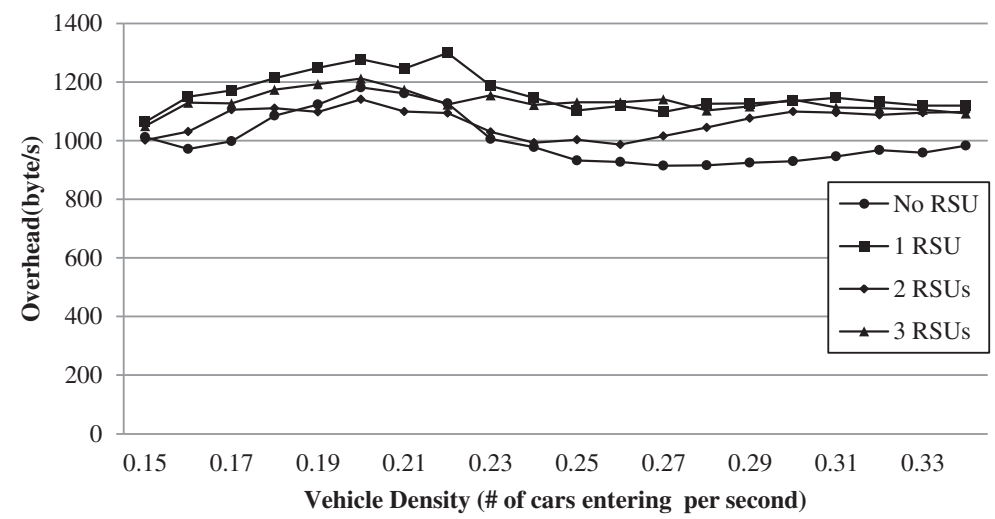

(a)

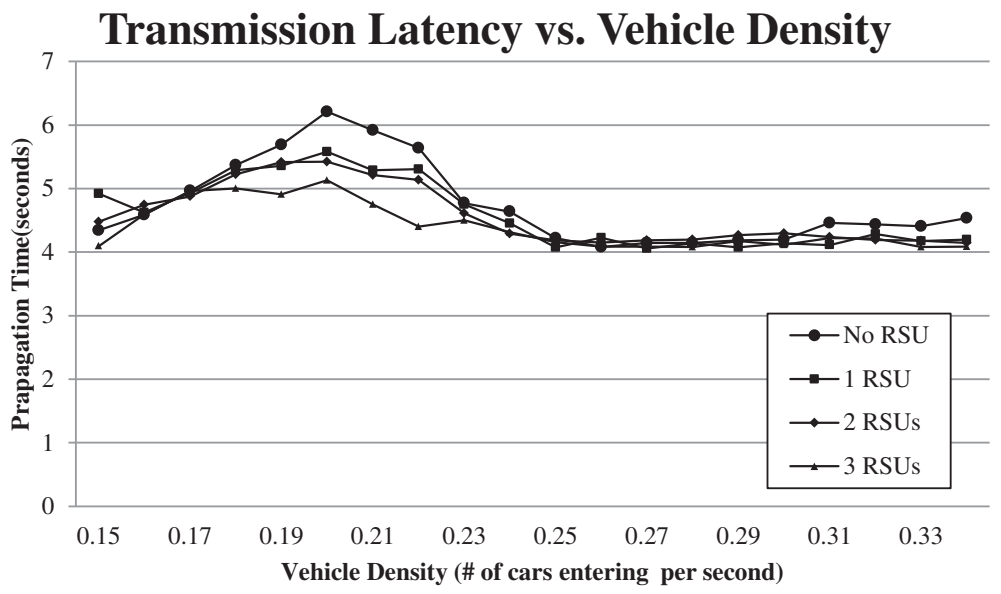

(b)

Figure 7 The results of model I. (a) The impact of the vehicle density on the signaling overhead. (b) The impact of the vehicle density on the propagation time of ABMs. 
distances between cars and the hop distance decrease as the rate increases. However, when the density increases further, the distance between adjacent cars becomes small enough for the transmission range to include the second car, so the hop distance increases again. The simulation results show that it takes less than $7 \mathrm{~s}$ for messages to travel $2 \mathrm{~km}$. For non-urgent data, this speed is quite acceptable.

\subsection{Model II}

In model II, we compare LOA-CAST's performance with that of traditional flooding broadcast approaches. For flooding, the message size is set the same as that of the $\mathrm{ABM}$, and the broadcast is identical to $T_{\text {period. }}$ Figure $8 \mathrm{a}$ shows the signaling overheads of the compared methods. The overhead increases as the vehicle density grows because ABMs can be forwarded over a broader range more frequently. However, the overhead saturates if the density is even higher. Compared to conventional flooding approaches, the signaling overhead of LOA-CAST is very light. Since information is placed into the ABM in our proposed scheme, the signaling overhead does not vary as the number of information sources changes. On the other hand, the overhead of conventional flooding approaches grows as the number of sources increases because the messages of different sources are forwarded independently and are not aggregated. Even in the worst case, the overhead of LOA-CAST is lighter than the overheads of the two sources in the compared approaches. It is obvious that LOA-CAST is a reasonable broadcasting approach with a lightweight overhead. Figure $8 \mathrm{~b}$ shows the proportion of beaters. As the vehicle density increases, the proportion of beaters decreases because when there are fewer cars, it is more difficult for each car to find a forward car. Therefore, more cars switch to the beating mode. However, the proportion gradually saturates as the density increases further. This fact demonstrates that when the vehicle density is at a certain level, the operation

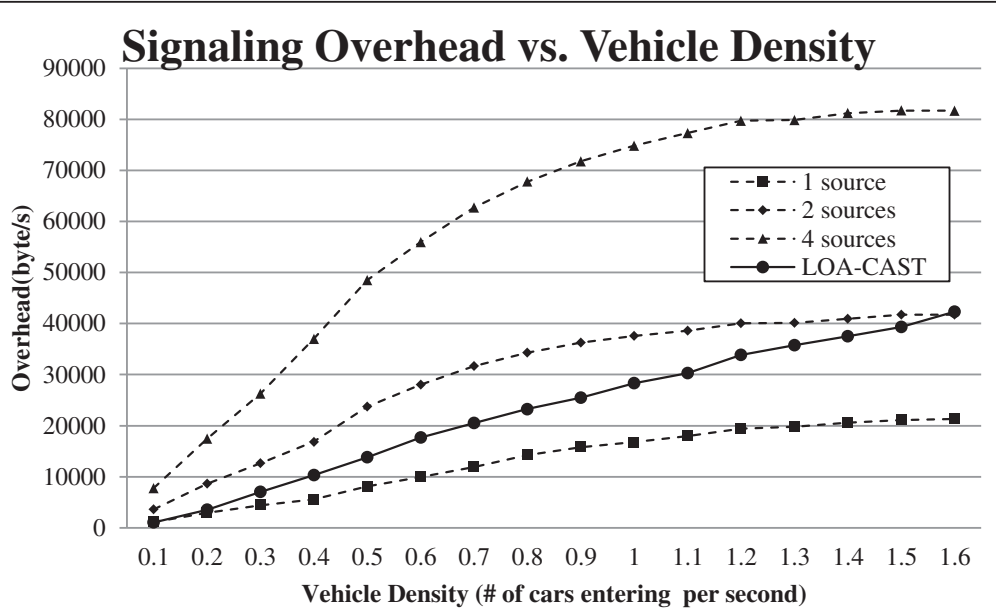

(a)

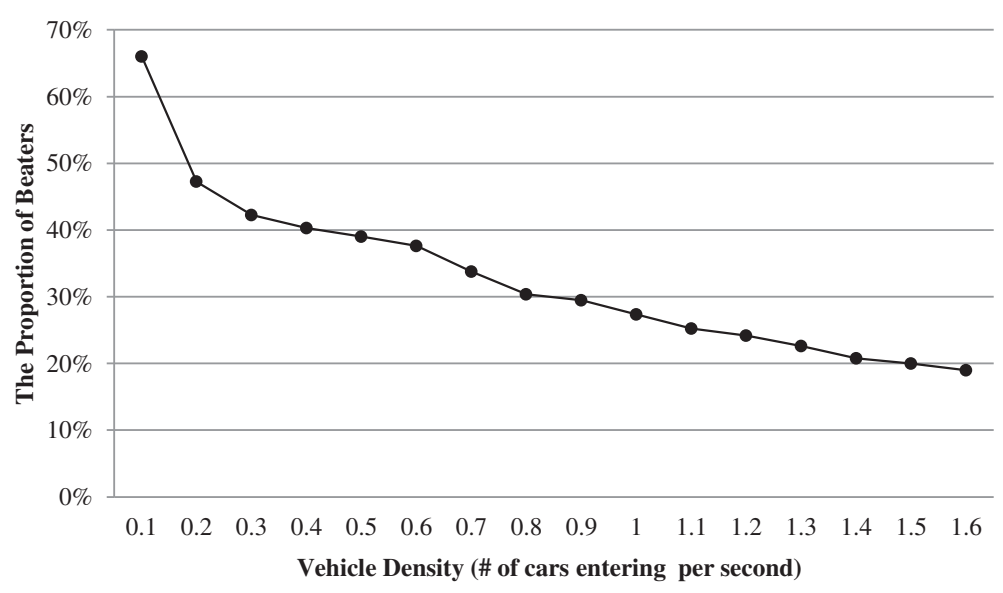

(b)

Figure 8 The results of model II. (a) Signaling overheads of the compared methods. (b) The proportion of beaters. 
of LOA-CAST is stable, which correlates with the overhead trend in Figure 8a.

\section{Conclusion}

In this paper, we have proposed a message aggregation scheme, called LOA-CAST, for non-urgent broadcasts in VANETs. The scheme aggregates the payloads of different information sources and provides a framework that allows messages to propagate. Because of each node's distributed operation, LOA-CAST's signaling overhead is light, while the transmission range of each information source is adaptable to the current traffic load. Our analysis shows that the scheme is robust and stable when it is attacked by malicious/malfunctioning nodes. The simulation results also show that in different scenarios, LOA-CAST's performance is stable, its signaling overhead is reasonable, and its transmission speed is acceptable.

\section{Competing interests}

The authors declare that they have no competing interests.

Received: 8 January 2014 Accepted: 26 March 2014

Published: 10 April 2014

\section{References}

1. H Hartenstein, K Laberteaux, A tutorial survey on vehicular ad hoc networks. IEEE Commun. Magazine 46(6), 164-171 (2008)

2. IEEE 802.11 Standard, IEEE-SA, http://standards.ieee.org/getieee802/ download/802.11-2012.pdf. Accessed 5 April 2012

3. B Karp, HT Kung, GPSR: Greedy perimeter stateless routing for wireless networks, in Proceedings of the ACM/IEEE international conference on mobile computing and networking (MobiCom). Boston, 6-11 August 2000

4. C Lochert, H Hartenstein, J Tian, D Herrmann, H Füßler, M Mauve, A routing strategy for vehicular ad hoc networks in city environments, in Proceedings of IEEE intelligent vehicles symposium (IV2003). Columbus, OH, USA, 9-11 June 2003, pp. 156-161

5. CR Lin, M Gerla, Adaptive clustering for mobile wireless networks. IEEE J. Selected Areas in Commun. 15(7), 1265-1275 (1997)

6. J Wu, H Li, A dominating-set-based routing scheme in ad hoc wireless networks. The special issue on Wireless Networks in the Telecommunication Systems Journal 3, 63-84 (2001)

7. MD Dikaiakos, A Florides, T Nadeem, L Iftode, Location-aware services over vehicular ad-hoc networks using car-to-car communication. IEEE J. Selected Areas in Commun. 25, 1590-1602 (2007)

8. M Torrent-Moreno, D Jiang, $\mathrm{H}$ Hartenstein, Broadcast reception rates and effects of priority access in 802.11-based vehicular ad-hoc networks, in Proc. ACM ICMCN. New York, NY, USA 1 October 2004

9. J Zhao, Y Zhang, G Cao, Data pouring and buffering on the road: a new data dissemination paradigm for vehicular ad hoc networks. IEEE Trans. Vehicular Technol. 56, 3266-3277 (2007)

10. O Tonguz, N Wisitpongphan, F Bai, P Mudalige, V Sadekar, Broadcasting in VANET, in Proc. IEEE Mobile Networking for Vehicular Environments. Anchorage, AK, 11 May 2007

11. C Maihöfer, A survey of geocast routing protocols. IEEE Commun. Surv. Tutorials 6(2), 32-42 (2004)

12. C Maihöfer, R Eberhardt, Geocast in vehicular environments: caching and transmission range control for improved efficiency, in Proc. IEEE Intelligent Vehicles Symposium. Parma, Italy, 14-17 June 2004

13. C Maihöfer, T Leinmüller, E Schoch, Abiding geocast: time-stable geocast for ad hoc networks, in Proceedings of the 2nd ACM International Workshop on Vehicular Ad Hoc Networks (VANET'05). New York, NY, USA, 2 September 2005, pp. 20-29
14. K Ibrahim, M Weigle, Accurate data aggregation for VANETs, in Proceedings of the 4th ACM International Workshop on Vehicular Ad Hoc Networks. Montreal, Canada, 9-14 September 2007, pp. 71-72

15. S Dietzel, On the potential of generic modeling for VANET data aggregation protocols, in Proceedings of Vehicular Networking Conference. Hyatt Jersey City, NJ, USA, 13-15 December 2010, pp. 78-85

doi:10.1186/1687-1499-2014-56

Cite this article as: Kuo and Wu: LOA-CAST: a novel low-overhead information broadcast scheme for vehicular ad hoc networks. EURASIP Journal on Wireless Communications and Networking 2014 2014:56.

\section{Submit your manuscript to a SpringerOpen ${ }^{\circ}$ journal and benefit from:}

- Convenient online submission

- Rigorous peer review

- Immediate publication on acceptance

- Open access: articles freely available online

- High visibility within the field

- Retaining the copyright to your article

Submit your next manuscript at $>$ springeropen.com 